\title{
Study Designs in Medicine
}

Scientific studies can be described as " $a$ planned and systematic effort based on evidence for the solution of any health problems using data with high degree of accuracy" (1). The main aims are to quantify disease prevalence, and compare interventions, predictions, association assessments or etiology assessments (2). A scientific study requires good planning including research protocol, ethical approval, data collection, data analysis, interpretation of data analysis results and publication. This study can help authors understand study designs in medicine.

Scientific studies can be classified as "Basic Studies", "Observational Studies", "Experimental (Interventional) Studies", "Economic Evaluations" and "Meta-Analysis - Systematic Review", as shown in Figure 1.

\section{BASIC STUDIES}

Basic studies investigate the cause-outcome relationships between a dependent variable and independent variables, such as animal experiment, genetic and cell studies. Also, method development studies investigate the development and improvement of biochemical (e.g., enzymes, markers or genes), imaging (e.g., magnetic resonance) and biometric methods (e.g., statistical methods) (3). Several checklists have been developed to guide authors in the preparing, con- ducting and reporting stages of their studies. The ARRIVE checklist supplies transparency and accuracy in the animal experiments (1).

\section{OBSERVATIONAL STUDIES}

Observational studies can be defined as non-interventional and non-experimental (3). They do not contain any experiment or intervention methods. Investigated factors aren't controlled, repetition of events aren't generally possible and randomisation facilities are limited in these studies. However, their results are largely consistent with real life (4). They can be classified as descriptive or analytical, as shown in the Figure 1.

\section{Descriptive studies}

Health problems or events as regards a particular disease or condition are detected and identified in these studies. They seek answers to the following questions about health problems or events: "What is it?", "Where is it seen?", "When is it seen?" and "Who are observed?" Descriptive statistics (mean, rate, etc.), frequency distributions and population parameters are determined by this kind of research.

Descriptive observational studies include case-report, case series and cross-sectional studies (descriptive or prevalence).

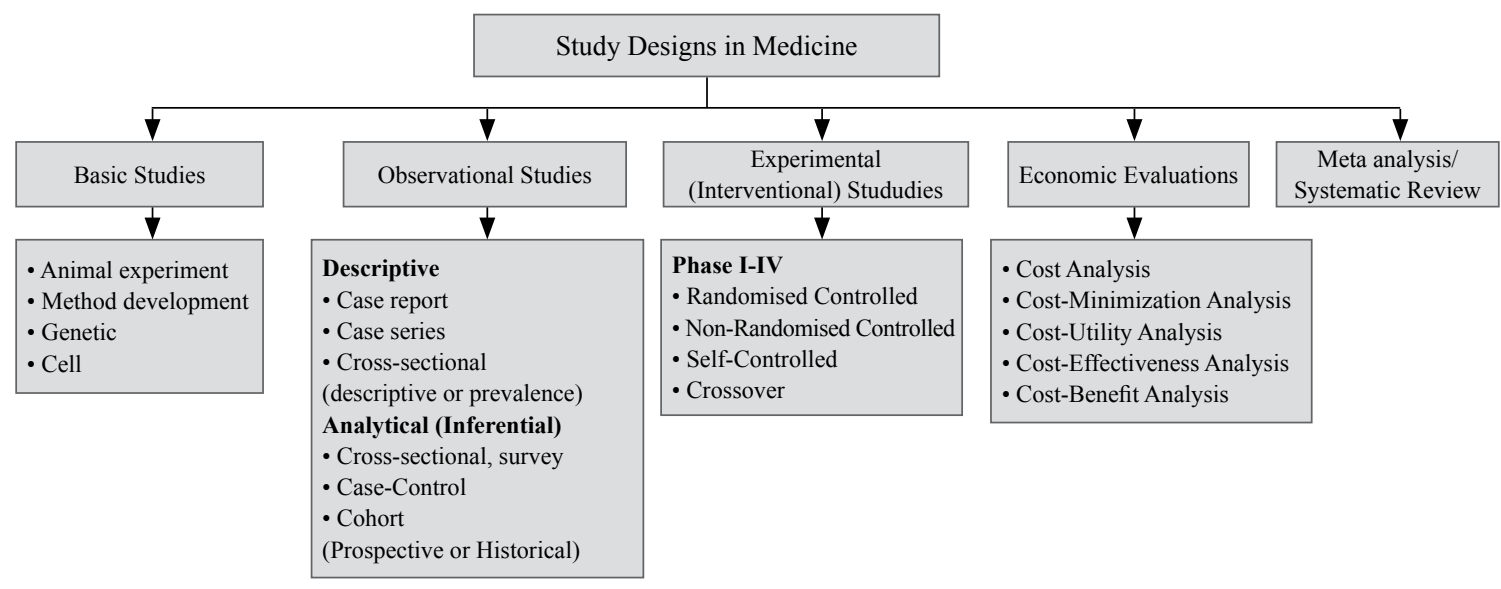

FIG. 1. Study designs

\author{
Address for Correspondence: Dr. Necdet Süt, Department of Biostatistics and Informatics, Trakya University Faculty of Medicine, Edirne, Turkey \\ Phone: +902842357641 e-mail: nsut@trakya.edu.tr \\ - DOI: 10.5152/balkanmedj.2014.1408 \\ Available at www.balkanmedicaljournal.org \\ Cite this article as: \\ Süt N. Study designs in medicine. Balkan Med J 2014;31:273-7.
}


Patient and disease characteristics related to some interesting and remarkable type defined in a patient are called a "case report". When the number of patients is more than one, this is called a "case series". These are the most simple research types and do not contain a control group. Case series are usually starting points of the examined hypothesis in the casecontrol, cross-sectional or cohort studies (5). The use of CARE statement in the publication of a case report supplies transparency and accuracy (6).

Cross-sectional studies (descriptive or prevalence) can be described as prevalence studies and generally examine the prevalence, epidemiology or survey of a disease or clinical outcome. They reflect the situation of a disease or clinical outcome at a particular moment in a particular population (5).

\section{Analytical (inferential) studies}

\section{Cross-sectional study}

Analytical cross-sectional studies are conducted in a specific time period which does not contain follow-up and enquires: "What is happening in a specific time period?" (Figure 2) They try to explain potential causal associations between causes (exposures) and outcome (disease or clinical outcome). As a cohort study, they compare disease prevalence between exposure groups, and as a case-control study, they compare exposure between disease and healthy groups (2). Generally,

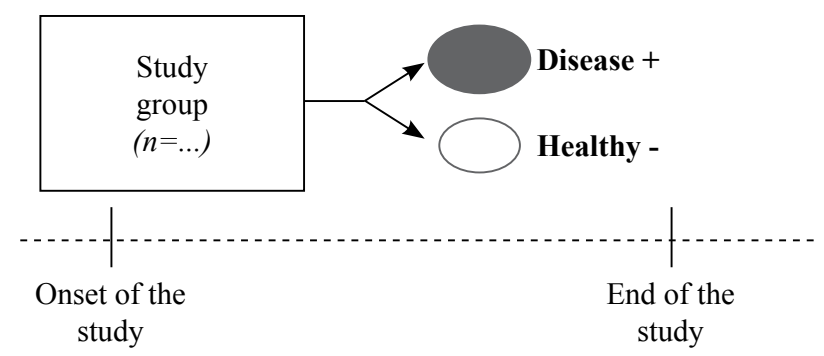

FIG. 2. Cross-sectional study design

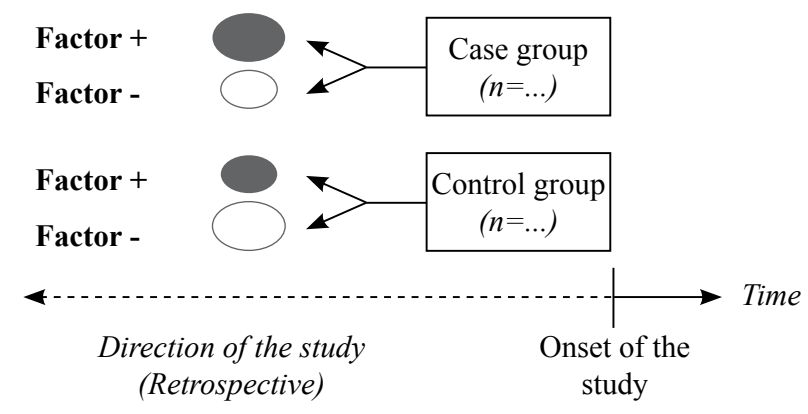

FIG. 3. Case-control study design they do not have a follow-up period.(5) Checklists guide the authors in preparing, conducting and reporting stages of research. The STROBE statement for cross-sectional studies is a useful guideline for this design (1).

\section{Case-control study}

Case-Control Studies are conducted retrospectively and enquire: "What happened in the past?" (Figure 3). The cases are subjects selected according to presence of disease or clinical outcome. However, the control subjects are selected without disease or clinical outcome. The case and control groups are compared in terms of the presence of certain factors. Case group should be matched to the control group except for investigated factors. These are matched case-control study (5). The STROBE statement for case-control studies guides authors (1).

Diagnostic Accuracy Studies investigate the effect of a diagnostic method (such as imaging, pathological) compared with a gold standard method (3). They are similar to case-control studies. The STARD statement helps authors in designing, conducting and reporting diagnostic accuracy studies (1).

\section{Cohort study}

Cohort is a special group of people who have been selected according to some defining characteristics and they have certain disease risk factors or health outcome. Cohort Studies, also called follow-up studies, are generally prospective and enquire: "What will happen in the future?" (Figure 4) Individuals are followed over time in cohort studies, and researchers assess exposure and outcome during follow-up (2). Cohort studies investigate the effect of prognostic factors (such as age, presence of hypertension and cholesterol level) on a clinical outcome (such as disease) (3).

Moreover, cohort studies can be conducted retrospectively; these are called "Historical Cohort Study". Cohort Studies produce the most reliable clinical evidence among the observational studies due to the fact that they identify clinical or

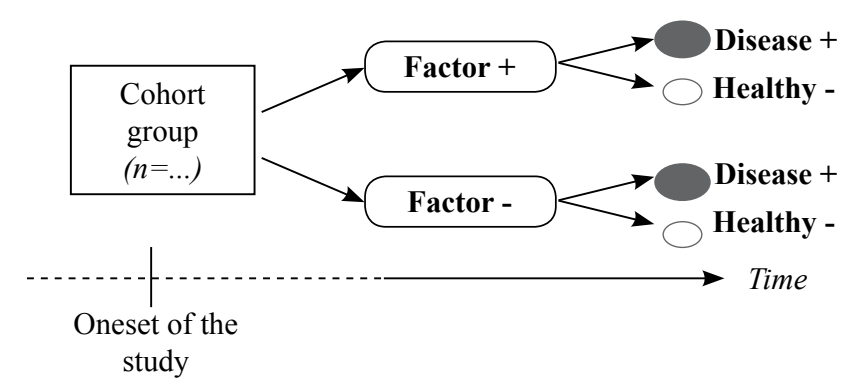

FIG. 4. Cohort study design 
health outcomes based on exposure (5). The STROBE statement for cohort studies helps authors (1).

\section{EXPERIMENTAL (INTERVENTIONAL) STUDIES}

Experimental or interventional studies compare the effect of treatments or interventions with control in humans. Placebo or different treatment(s) or intervention(s) may be used as control. Experimental studies have to be transparent and evidence-based. In these studies, randomisation methods can be used, investigated factors are controlled, cause-effect relationships are evidenced and an experiment can be repeated as much as desired. However, their results are always not appropriate for real life (4). They can be conducted in four phases (7).

Phase I study is conducted in a small number of healthy volunteers (e.g. 20-80) to determine whether a drug or treatment method is safe. Pharmacokinetic and pharmacodynamic measurements are done in these studies. Maximum safe dose, movement of the drug in the body and dose-response relationship are examined. Phase II study is conducted in a target population (75-300) to determine the treatment effect of a drug or treatment method. Standard treatment method has to be compared with placebo in Phase II clinical trials. Phase III study is conducted on many patients (e.g., 1000-2000) to determine whether the new drug is better than the standard drug. It is done in order to reveal that a drug is not only safe and effective, but also has better and less adverse effects than standard treatment. Usually, at least two RCTs are required in this phase.

Clinical trials (Phase IV) are called post-marketing product surveillance studies, which are conducted on patients in daily life; the new drug had been approved by the Ministry in this phase. They evaluate the adverse effect and various additional indications of a new drug $(7,8)$.

Observational Drug Studies are other forms of Phase IV clinical trials. They collect the data about a spontaneously prescribed drug from the patients with diagnosed and ongoing treatment. In these studies, additional information from a larger population may be obtained in order to compare the results of experimental clinical drug trials (4).

\section{Randomised controlled trial (RCT)}

Randomised controlled trials produce the strongest evidence among clinical trials due to the fact that patients are allocated to treatments or interventions randomly (equal chance). In these studies, two or more clinical treatments or intervention are compared. RCTs are expensive and slow, however, their level of evidence is higher due to the fact that randomisation removes the allocation bias (2). Many respected journals en- dorse the CONSORT statement in order to improve the scientific quality and transparency of RCTs. Authors should be used to the CONSORT statement as a guideline in RCTs (1).

When the preference of participants is not to receive a placebo or control, randomisation procedure is not applied. These studies are called Non-Randomised Controlled Studies. They are inexpensive especially if they are conducted as retrospective and representative sample of patients in clinical practice. However, they are open to bias (2).

\section{Self-controlled study}

Self-Controlled Studies do not include an independent control group; they use the patients as their own controls. At least two measurements are obtained at different times from the same patients (e.g., preop, postop 1. month, and 6. month measurements) and the effect of treatment or intervention is determined (5).

\section{Crossover study}

Crossover Studies include both of self-control and independent groups. They are powerful, but not always possible to apply. In crossover studies, patients are assigned two groups (placebo or experimental treatment). After a time, the research is interrupted for a washout period (at least two weeks), and patients receive no treatments during this period. At the end of the washout period, the experimental treatment group receives the placebo and the placebo group receives to the experimental treatment (5). The effect of treatment or intervention is determined by comparisons of both self-control and independent groups in crossover design (Figure 5).

\section{Properties of experimental studies}

\section{Direction of studies}

Studies can be classified as prospective or retrospective according to direction. In prospective studies, a specific sample is followed over a certain period in order to determine outcome from the reasons. The research question is: "What will happen in the future?" Retrospective studies generally compare the outcome of diagnostic and treatment methods. Data

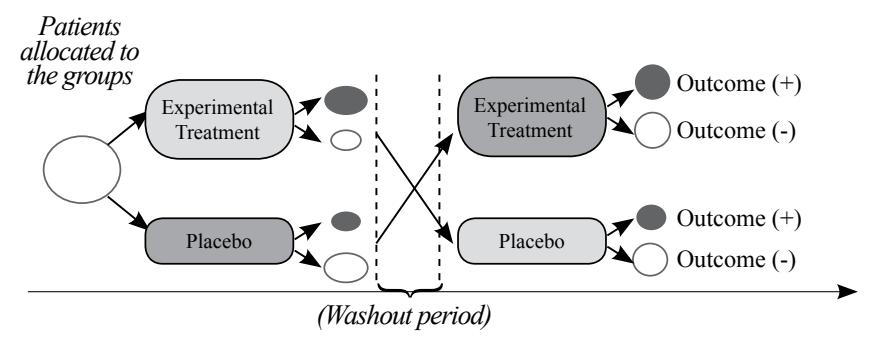

FIG. 5. Crossover study design 


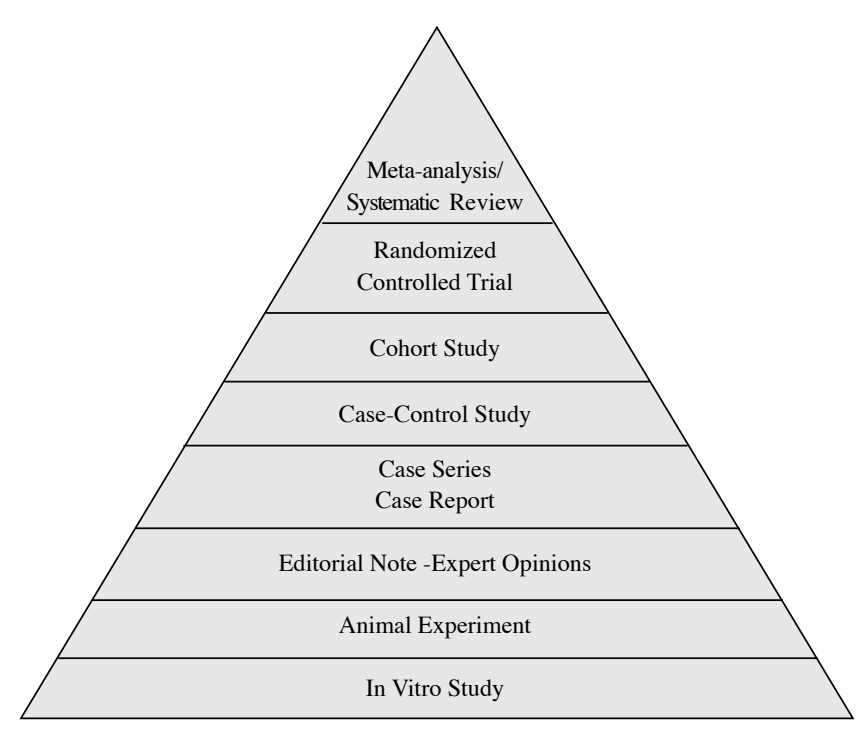

FIG. 6. Evidence pyramid for medical studies

are obtained from patient records. The research question is: "What happened in the past?" (5).

\section{Randomisation}

In randomisation method the subjects or patients who will be included in the study are assigned to treatment groups with equal probability (chance) in the beginning of study. A computerized software is widely used for allocating the subjects/ patients to the groups in the randomisation processes. Studies can be classified as i) randomised or ii) non-randomised. Randomised Controlled Studies (RCTs) produce the most reliable results among all research types.

\section{Blinding}

Blinding describes that one or more of the physicians, researchers, patients and data analysts do not know which treatment subjects have received. It ensures reliable and objective results preventing bias. Blinding can be defined as three different types (single, double and triple). Single-blind: either subjects or researchers know which treatment subjects have received. Double-blind: both subjects and researchers do not know which treatment subjects have received. Tripleblind: in addition to the subjects and researcher(s), statisticians/monitors do not know which treatment subjects have received (5).

\section{Confounding and interaction}

Confounding can be defined as disruption of the relationships between two variables due to the effect of third variable. A confounder variable is associated both with causal and out- come variables (9). Two or higher independent variables have different effect on outcome variable to independent effect of each. This situation can be defined as interaction.

\section{ECONOMIC EVALUATIONS}

Cost Analysis is an economic analysis method that estimates total cost of a particular disease or health condition on society. Direct and indirect costs attributed to a specific disease are included in this method. It is also called "cost of illness". Cost-Minimisation Analysis compares two alternative drugs' (or interventions) costs and outcomes in order to determine the least costly drug (or intervention). However, it is quite difficult to find two alternative drugs which are equally effective and safe. Thus, it is rarely used in economic evaluations. Cost-Utility Analysis is an economic evaluation method comparing two alternative drugs (or interventions) costs and outcomes in order to determine the most useful drug (or intervention). Outcomes in these studies are measured in preference or utility of patients, and, generally, quality-adjusted life year (QALY) or disability-adjusted life year (DALY) are used as an outcome. Cost-Effectiveness Analysis compares two alternative drugs (or interventions) costs and clinical outcomes in order to determine the most effective drug (or intervention). Outcomes are measured by clinical parameters. It is the most widely used economic evaluation method. Cost-Benefit Analysis is an economic evaluation method, in which cost and benefit of alternative interventions are expressed in monetary units. Thus, it is rarely used in economic evaluations (8).

\section{META-ANALYSIS AND SYSTEMATIC REVIEW}

Several clinical studies (RCTs or Cohort) may be conducted in a clinical area over a period of years in different parts of the world. The results may be different and there may be different properties such as sample size and multicentre. Meta-Analysis combines the statistical results of different studies in a particular clinical area $(7,9)$. The PRISMA statement guides the authors in the preparation of Meta-Analysis (1).

Systematic Review evaluates and interprets the evidence of all studies conducted in a clinical area (9). The main difference from Meta-Analysis is that it combines the evidence of different studies based on interpretation instead of combining statistical results.

\section{Evidence level of the medical studies}

The evidence pyramid shows the evidence level of a scientific study in clinical practices. The evidence pyramid of sci- 
entific medical studies is shown in Figure 6. According to the evidence pyramid, the "Meta-Analysis/Systematic Review" produces the most reliable evidence, while "in vitro study" produces the lowest reliable evidence (10).

In conclusion, authors should correctly report the study design in the method section of their studies. Also, if randomisation, stratification or blinding methods are used, they should be reported in this section. Generally, studies are conducted on a sample, so sample size should be a sufficient number and representative of population in structural terms. Thus, determination of sample size, selection method of sample, inclusion and exclusion criteria should be explained in detail in the method section. Use of the checklists (CONSORT statement for RCTs, ARRIVE for animal experiments and STROBE statement for cross-sectional, case-control and cohort studies, CARE statement for case report and PRISMA statement for meta-analysis) may prevent bias and guide authors in the preparation of their studies.

\section{REFERENCES}

1. Sut N. How can we improve the quality of scientific research and publications? Guidelines for authors, editors, and reviewers. Balk Med J 2013;30:134-5. [CrossRef]
2. Altman D. Introduction to study design 2014 [14.10.2014]. Available from: http://www.equator-network.org/wp-content/uploads/2014/10/ Introduction-to-study-design-Doug-Altman.pdf.

3. Rohrig B, du Prel JB, Wachtlin D, Blettner M. Types of study in medical research: part 3 of a series on evaluation of scientific publications. Dtsch Arzteblatt Int 2009;106:262-8.

4. İskit AB. İlaçlarla yapılan gözlemsel (farmakoepidemiyolojik) çalışmalar kılavuzu. İKU Dergisi 2008;20:25-33.

5. Dawson B, Trapp RG. Basic and clinical biostatistics. New York: Lange Medical Books/McGraw-Hill; 2004.

6. Gagnier JJ, Kienle G, Altman DG, Moher D, Sox H, Riley D, et al. The CARE guidelines: consensus-based clinical case report guideline development. J Clin Epidemiol 2014;67:46-51. [CrossRef]

7. Friedman LM, Furberg CD, DeMets DL. Fundamentals of clinical trials. New York: Springer-Verlag; 1998. [CrossRef]

8. Berger ML, Bingefors K, Hedblom EC, Pashos CL, Torrance GW. Healt care cost, quality, and outcomes. NJ: ISPOR; 2003.

9. Hunink M, Glasziou P. Decision making in health and medicine. Cambridge: Cambridge University Press; 2001.

10. Pandis N. The evidence pyramid and introduction to randomized controlled trials. Am J Orthod Dentofacial Orthop 2011;140:446-7. [CrossRef]

\section{Necdet SÜT, PhD \\ Biostatistics Editor of Balkan Medical Journal \\ Department of Biostatistics and Medical Informatics, Trakya University Faculty of Medicine, Edirne, Turkey}

